\title{
Combination of Q-Switched Nd:YAG and Fractional Erbium:YAG Lasers in Treatment of Melasma: A Randomized Controlled Clinical Trial
}

\author{
Shiva Alavi ${ }^{1,2}$, Ehsan Abolhasani ${ }^{2}$, Sharin Asadi ${ }^{2}$, Mohammadali Nilforoushzadeh ${ }^{2 *}$ \\ ${ }^{1}$ Skin Diseases and Leishmaniasis Research Center, Isfahan University of Medical Sciences, Isfahan, Iran \\ ${ }^{2}$ Skin and Stem Cell Research Center, Tehran University of Medical Sciences, Tehran, Iran
}

\author{
*Correspondence to \\ Mohammad-Ali Nilforoushzadeh \\ MD; Skin and Stem Cell Research \\ Center, Tehran University of \\ Medical Sciences, Tehran, Iran. No \\ 4, Maryam Dead-end Alley, South \\ Kamraniyeh Street, Tehran, Iran. \\ Postal code: 1417653761 . \\ Tel: $+98-2122674977$ \\ Fax: +98-2122239264 \\ Email: dr_nilforoush@yahoo.com
}

Published online 8 January 2017

\begin{abstract}
Introduction: Ablative and nonablative lasers have been used to treat melasma. We aimed to assess and compare the combining Q-switched Nd:YAG laser (QSNYL) and fractional erbium:YAG laser (FEYL) with QSNYL alone in treatment of melasma.

Methods: This randomized controlled clinical trial was performed in our Research Center during 2013-2014. Women with melasma and without a history of keloid formation, hypersensitivity to hydroquinone, or pigmentary changes due to laser therapy were randomly allocated to receive four sessions of either QSNYL-FEYL combination or QSNYL alone. All patients received topical treatment with Kligman's formula. Before laser therapy and 4 weeks after the last treatment session, patients' skin was assessed for changes in skin color, melanin content, and erythema intensity of melasma lesions quantitatively.

Results: Finally, 21 patients in QSNYL-FEYL and 20 in QSNYL group (mean age, 38.57 [5.60] and 42.60 [8.44] years, respectively) completed study. The skin color had become lighter in both groups (mean [SD] percentage change of 56.95 [40.29] and 29.25 [13.20] in QSNYL-FEYL and QSNYL groups, respectively) with significantly better results in QSNYLFEYL group $(P=0.006)$. Percentage of decrease of melanin content was significantly higher in QSNYL-FEYL group (22.01 [10.67] vs. 7.69 [4.75]; $P<0.001)$. After adjustment for baseline values, the post treatment intensity of erythema was significantly lower in QSNYLFEYL group $(P<0.001)$. The patients reported no adverse events.

Conclusion: QSNYL-FEYL was significantly more effective in decreasing melanin content of lesions than QSNYL and led to a lighter skin.

Keywords: Melasma; Melanosis; Lasers, Solid-state; Erbium-doped yttrium aluminum garnet lasers; Neodymium-doped yttrium aluminum garnet lasers.
\end{abstract}

\section{Introduction}

Melasma is the most common pigmentary skin disorder, which is seen more frequently in females and those with darker complexion. Many factors contribute to the development of melasma including ultraviolet exposure, pregnancy, oral contraceptive pills, endocrine and hormonal factors, and genetic predisposition. It is a chronic and hard-to-treat disorder and although different therapeutic modalities have been employed to treat the disorder, ${ }^{1}$ search for an effective method of therapy continues.

Nonablative laser, alone or in combination with ablative lasers and topical medications, have been employed to treat pigmentary disorders including melasma. Nonablative lasers such as Q-switched Nd:YAG laser (QSNYL) that selectively target melanosomes ${ }^{2-4}$ and ablative lasers such as fractional erbium:YAG laser (FEYL) or fractional $\mathrm{CO}_{2}$ laser (FCOL) have been used frequently for this purpose. ${ }^{5}$ Nonetheless, we could not find any study that had employed both laser systems in treatment of melasma. Moreover, most of the studies have used melasma area and severity index (MASI) or physician's global assessment (GPA) to evaluate the improvement of therapy; however, objective assessment of the melanin content and changes in lesions' color would provide more reliable result in terms of treatment efficacy.

Although patients with melasma were treated with either QSNYL or FEYL in our clinic, combining these two systems had not been tried before. We aimed to assess the efficacy of combination laser therapy with QSNYL and FEYL in treatment of melasma.

\section{Methods}

This randomized, controlled, assessor-blinded clinical trial was conducted in our Research Center during 20132014. 


\section{Patients}

Patients at reproductive age with melasma of the malar region and nose were included. Patients with active lesions or infection in treatment area, history of keloid formation, hyperpigmentation after laser therapy, hypersensitivity to hydroquinone, or any condition that would hamper patients' participation were excluded. Patients were informed of study protocol and possible benefits and adverse effects. A written informed consent was obtained from those who met the eligibility criteria and accepted to participate in the study.

\section{Sample Size}

Most of the studies in this field were case series, which had made calculating sample size difficult. With regard to the study by Niwa Massaki et $\mathrm{al}^{6}$ and by considering $\alpha$ of 0.05 , study power of $95 \%$, and using the following formula, the sample size was calculated at 21 in each group:

$$
n=\frac{\left(z_{1-\frac{a}{2}}+z_{1-\beta}\right)^{2}\left(\delta_{1}^{2}+\delta_{2}^{2}\right)}{\left(\mu_{1}-\mu_{2}\right)^{2}}
$$

$\left(Z_{1-\alpha / 2}=1.96, Z_{1-\beta}=1.64, \delta_{1}=5.4, \delta_{2}=4.4, \mu_{1}=13.2\right.$, and $\left.\mu_{2}=7.7\right)$

\section{Randomization and Blinding}

We used random-sequence blocks with size of four (ie, $\mathrm{AABB}, \mathrm{BBAA}, \mathrm{ABAB}, \mathrm{BABA}, \mathrm{ABBA}$, and $\mathrm{BAAB})$ to allocate participants randomly to receive QSNYL and then FEYL (QSNYL-FEYL group) or only QSNYL therapy (QSNYL group). Blinding the patients and therapist was not possible as one group received therapy with one laser system and the other with 2 different laser systems. Nonetheless, the physician in charge of assessing lesions through biometric devices and the statistician in charge of data analysis and reporting results were blinded to the assigned treatment to each patient.

\section{Interventions}

Before laser therapy, patients were instructed to apply Kligman's formula ( $0.1 \%$ tretinoin, $5.0 \%$ hydroquinone, and $0.1 \%$ dexamethasone in a hydrophilic ointment $)^{7}$ on their face nightly for at least 1 week before starting treatment and continue using it throughout treatment. Laser therapy sessions were hold every other week and each patient received four treatment sessions.

Half an hour before starting laser therapy, patients used $5 \%$ lidocaine/prilocaine cream (EMLA) on their face and covered the face with a sterile thin nylon. Few minutes before laser therapy, patients were instructed to cleanse their face with soap and water and dry it to remove any remaining cream.

Patients in QSNYL-FEYL group received QSNYL therapy with Hellios II system (Laseropole Co., Korea) with the following settings: Wavelength, $1064 \mathrm{~nm}$; energy, 400 to $500 \mathrm{~mJ}$; spot size, $8 \mathrm{~mm}$; and fluence, 0.769 to $0.995 \mathrm{~J} /$ $\mathrm{cm}^{2}$. After performing laser therapy, the patient's face was cooled down by putting icepacks on treated area. Thereafter, patients underwent FEYL treatment by Lotus II system (Laseropole Co., Korea) with the following settings: short mode; frequency, $10 \mathrm{~Hz}$; energy, $400 \mathrm{~mJ}$; spot size, $7 \mathrm{~mm}$; and fluence, $1.040 \mathrm{~J} / \mathrm{cm}^{2}$. Again, the patient skin was cooled down by icepack and was covered with hydrocortisone ointment and zinc oxide cream. Patients were instructed to apply a thermal water-based soothing cream (Cicalfate; Avene, Pierre-Fabre Group, France) for five days and then use Kligman's formula until the next laser therapy session. Patients were advised to wear sunscreen and avoid direct exposure to sunlight or any intense light. The treatment would be halted if the lesions' color would intensify.

Patients in QSNYL group only received QSNYL treatment with the same laser system and settings. Moreover, local anesthesia, treatments, and recommendations after laser therapy were the same for both groups.

\section{Outcome Measures}

To assess and compare the effects of treatment modalities, we employed two devices that quantified the changes in the skin. Mexameter MX 18 probe of C + K Multiprobe Adapter System (Courage + Khazaka Electronics, Cologne, Germany) measures skin melanin content as well as erythema via light absorption/reflection. The output of the device was numbers that facilitated the comparison of melanin and erythema at baseline with the contents in the same points after therapy. We also selected some points in nonlesional skin to see whether any change would be seen in nonlesional skin as the result of applied creams and to have a control for improvement of pigmentation in lesional skin. Visioface 1000 D (Courage + Khazaka Electronics, Cologne, Germany) was another device that helped achieve pictures of the face with the same light, distance, and magnification through inserting the head of patient in the cavity of device. The accompanying software helped to determine the changes in the skin color by measuring changes in different spots of the face. For each patient, we used the mean of four measurements as the final output of Visioface. Increase in the obtained value would indicate improvement of skin color, ie, getting lighter. Patients were assessed by Mexameter and Visioface half an hour before first laser session and four weeks after the last one.

\section{Statistical Analysis}

We used SPSS 16.0 (SPSS Inc., Chicago, IL, USA) to analyze the data. The data showed normal distribution in Kolmogorov-Smirnoff test and hence, parametric tests were employed. Paired-samples $t$ test was used to assess the changes from baseline in each group and differences between groups were evaluated by independent-samples $t$ test. The data were adjusted for confounding factors and analyzed through analysis of covariance (ANCOVA). For all tests, $P$ value $>0.05$ was considered statistically significant. 


\section{Results}

A total of 50 patients were evaluated and finally, 46 patients were enrolled in the study and randomly allocated to two groups of 23. Two patients in QSNYL-FEYL and three in QSNYL groups were lost to follow-up and the remaining entered the final analysis (Figure 1).

All patients were female and the means (SDs) of age in QSNYL-FEYL and QSNYL groups were 38.57 (5.60) (range, 30-49) and 42.60 (8.44) years (range, 25-57), respectively, with no significant difference between them $(P>0.05)$.

Both groups showed significant changes in mean values obtained from Visioface $(P<0.001$ for both groups) (Table 1 and Figure 2). There was no difference between two groups Visioface results after treatment $(P=0.197)$. The percent increase of Visioface score was significantly higher in QSNYL-FEYL group in comparison to QSNYL group (Table 2). Moreover, after adjusting for baseline value, the changes were more significant in QSNYL-FEYL group than in controls $(P<0.001$; Table 3$)$.

The changes in melanin content were compared before and after treatment in both melasma lesions and nonlesional skins. In QSNYL-FEYL group, both nonlesional and lesional skin showed significant decrease in mean melasma content; however the changes were more significant in lesional skin (laser-treated area) $(P<0.017$ and $P<0.001$, respectively). In QSNYL group, the melanin content was decreased in both nonlesional and lesional skin; however, the changes in nonlesional skin were not statistically significant. We also compared melanin content between the lesional and nonlesional skin of each patient. Although both groups showed significant differences between lesional and nonlesional skin at baseline, in contrary to patients in QSNYL group, patients in QSNYL-FEYL group showed no significant difference between melanin content of lesional and nonlesional skin after treatment (Table 1). Although the melanin content was significantly decreased in both groups, the decrease was significantly higher in QSNYLFEYL group (Table 2). There was no difference between two groups in melanin content changes in nonlesional skin before and after adjusting for baseline melanin content ( $P=0.915$ and $P=0.793$, respectively). On the other hand, after treatment, the melanin changes in lesional skin was significantly lower in QSNYL-FEYL group in comparison to controls, even after adjustment for baseline melanin content $(P<0.001$ in both) (Table 3$)$.

In QSNYL-FEYL group, there was no change in erythema intensity after treatment in nonlesional skin $(P=0.244)$ while it was significantly decreased in lesional skin $(P<0.001)$. In QSNYL group, no change was seen in erythema of nonlesional or lesional skin after treatment ( $P=0.08$ and $P=0.09$, respectively); nonetheless, there was a significant difference in erythema intensity between lesional and nonlesional skin at baseline as well as post treatment in both groups, ie, erythema was increased more profoundly in treated areas (Table 1). Although erythema intensity was decrease in both groups, there was no difference between groups in percent decrease of erythema (Table 2). After adjustment for baseline erythema, no difference was seen between two groups regarding changes in erythema of the nonlesional skin $(P=0.68)$ while QSNYL-FEYL group showed a significant decrease in erythema of lesional skin in comparison to

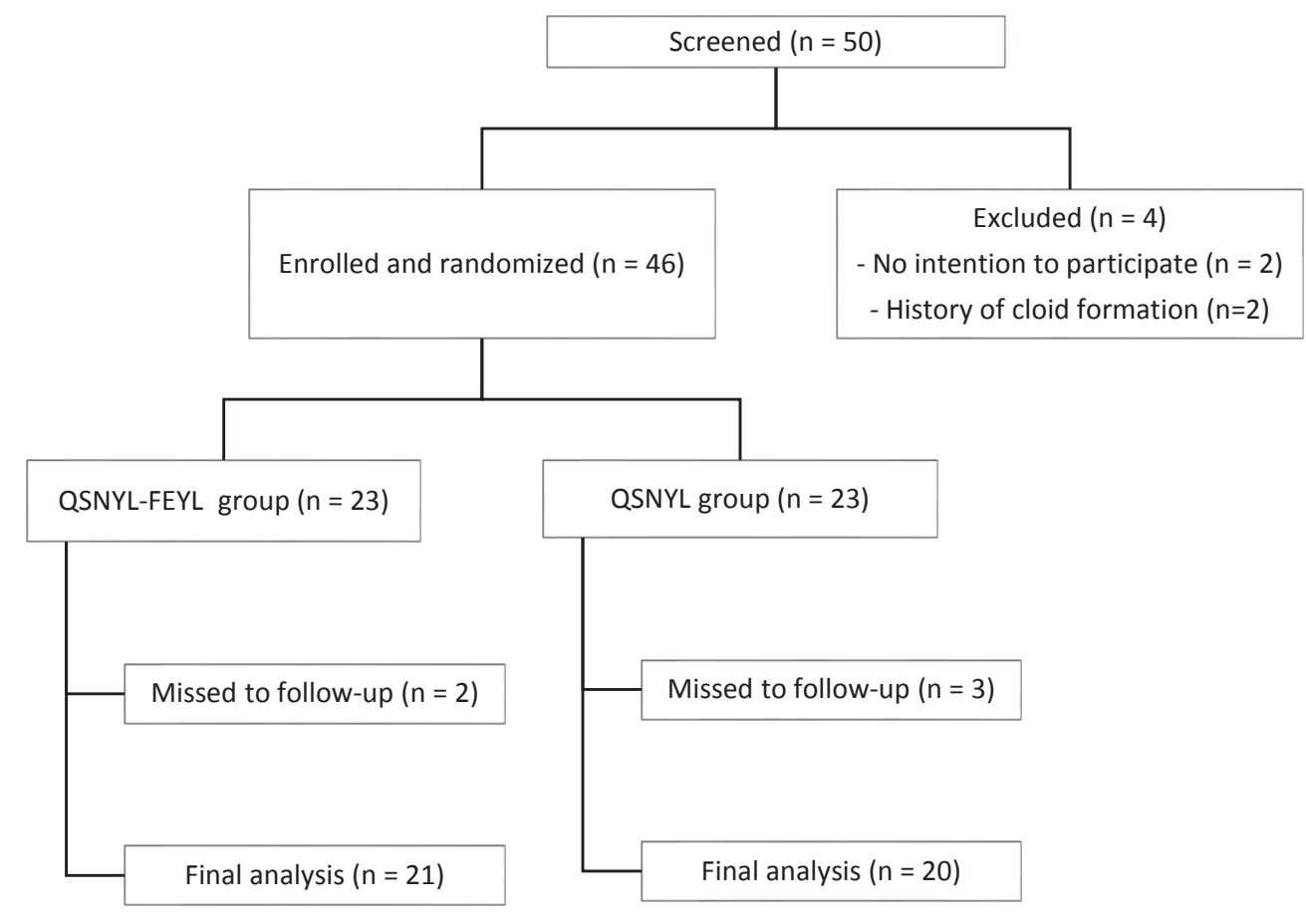

Figure 1. Flowchart of Enrollment Through Final Analysis.

Abbreviations: QSNYL, Q-switched Nd:YAG laser; FEYL, fractional erbium:YAG laser. 
Table 1. Visoface Scores, Melamine Content, and Erythema Intensity of Nonlesional and Lesional Skin in Study Groups ${ }^{\mathrm{a}, \mathrm{b}}$

\begin{tabular}{|c|c|c|c|c|c|c|}
\hline & \multicolumn{3}{|c|}{ QSNYL-FEYL Group $(n=21)$} & \multicolumn{3}{|c|}{ QSNYL Group $(\mathrm{n}=\mathbf{2 0})$} \\
\hline & Baseline & After Tx & $P$ Value & Baseline & After Tx & $P$ Value \\
\hline Visioface mean score & $-8.70(2.71)$ & $-3.57(2.87)$ & $<0.001$ & $-6.28(2.26)$ & $-4.64(2.25)$ & $<0.001$ \\
\hline Melanin, nonlesional skin & $158.95(38.02)$ & $154.95(36.43)$ & 0.017 & $160.92(7.06)$ & $156.11(7.28)$ & 0.050 \\
\hline Melanin, lesional skin & $220.57(39.70)$ & $170.42(31.42)$ & $<0.001$ & $226.85(49.88)$ & $209.75(48.99)$ & $<0.001$ \\
\hline $\begin{array}{l}P \text { value (comparing melanin content } \\
\text { between lesional and nonlesional skin) }\end{array}$ & $<0.001$ & 0.06 & - & $<0.001$ & $<0.001$ & - \\
\hline Erythema, nonlesional skin & $321.74(55.50)$ & $315.86(51.01)$ & 0.244 & $347062(62.98)$ & $333.30(59.10)$ & 0.078 \\
\hline Erythema, lesional skin & $349.03(62.53)$ & $320.47(43.72)$ & $<0.001$ & $393.05(34.22)$ & $378.55(37.11)$ & 0.09 \\
\hline $\begin{array}{l}\text { P value (comparing erythema between } \\
\text { lesional and nonlesional skin) }\end{array}$ & 0.011 & 0.002 & - & 0.002 & 0.002 & - \\
\hline
\end{tabular}

Abbreviations: QSNYL, Q-switched Nd:YAG laser; FEYL, fractional erbium:YAG laser; Tx, therapy.

a Data are presented as mean (SD).

${ }^{\mathrm{b}}$ Paired-samples $t$ test was used to compare the changes during treatment in each group. Independent-samples $t$ test was employed to compare values between two groups.

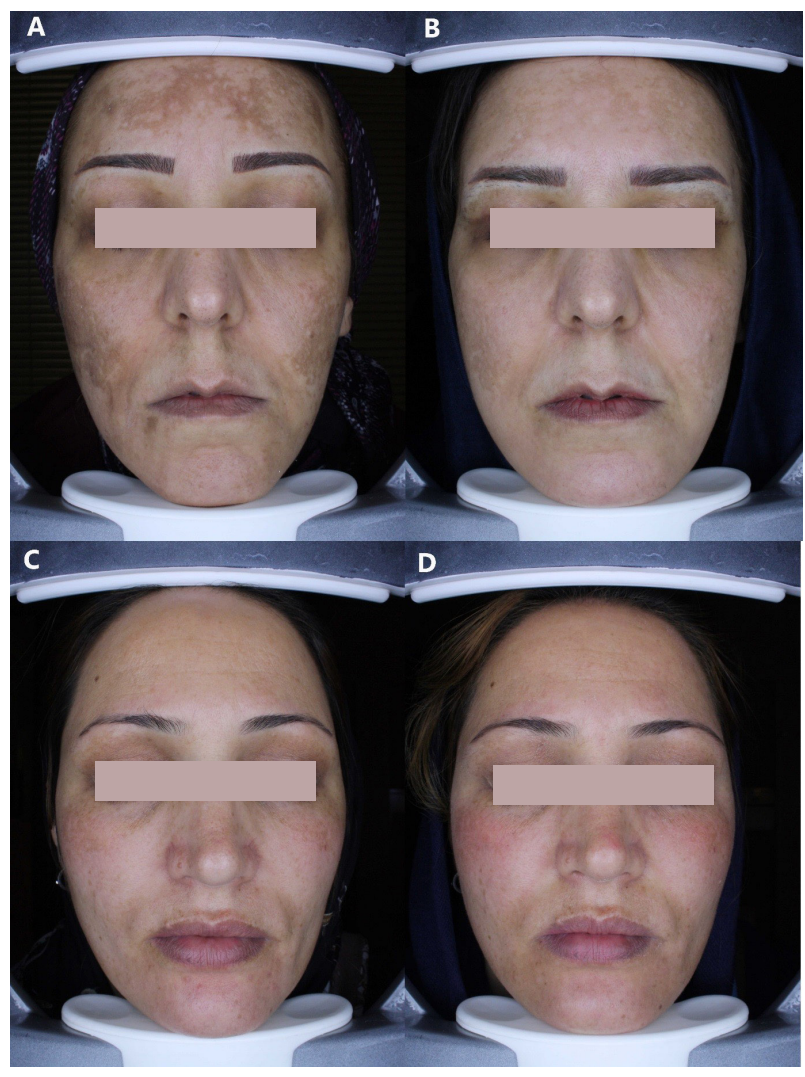

Figure 2. Comparing the Effect of Treatment by Visioface Photographs After 4 Sessions of Laser Therapy.

A 46-year-old woman with melasma of the forehead, malar region, and nose; $(\mathrm{A})$, before treatment with Q-switched Nd:YAG and fractional erbium:YAG lasers (mean score, -8.985); (B), four weeks after last session of laser therapy (mean score, -5.847).

A 32-year-old woman with melasma of the malar region and upper lids; (C), before treatment with Q-switched Nd:YAG (mean score, -6.52); (D), four weeks after last session of laser therapy (mean score, -5.255); erythema is evident at the site of laser therapy.

nonlesional skin $(P<0.001$; Table 3$)$.

While the safety of therapy was not among the outcome measures of our study, patients reported no adverse effect after completing the treatment; however, almost all of them had experienced two- to three-day self-limiting erythema after each laser session.

\section{Discussion}

We treated our patients with either QSNYL-FEYL or QSNYL and a topical administration of Kligman's formula. Treatment with both QSNYL-FEYL and QSNYL led to improvement in skin color; however, this improvement was more significant in QSNYL-FEYL group. Although the melanin content of lesions decreased in both group, the decrease was more significant in QSNYL-FEYL group, while demonstrating no significant difference in melanin content between nonlesional and lesional skin after treatment. Despite no significant difference in percentage of decrease of erythema intensity between groups, after adjustment for baseline value, erythema was significantly less intense in those treated with QSNYL-FEYL. In addition, patients of both groups reported no significant adverse effect of therapy. Only one patient withdrew from the study due to lack of subjective satisfactory results after three treatment sessions.

Ablative fractional lasers such as FCOL and FEYL, alone in combination with nonablative lasers such as Q-switched alexandrite laser (QSAL), were previously used to treat melasma lesions. In a case series, Nouri et al. employed FCOL and QSAL after 14 days of treatment with Kligman's solution to treat eight patients. In their study, one group received FCOL alone and the other received FCOL and then QSAL. They stated better results with combination therapy. ${ }^{8}$ In another split-face study, Angsuwarangsee et al compared the results of treatment with QSAL alone with that of QSAL and FCOL combination in six patients with refractory melasma and reported better results with combination therapy with regard to MASI score and melanin index. ${ }^{9}$

Although we could not find any study that had assessed the combination of QSEYL and FEYL, in treatment of melasma, both laser systems were employed previously and the results were compared with topical therapies. In 
Table 2. Percentage Changes of Visioface Score, Melanin Content, and Erythema Intensity of Nonlesional and Lesional Skin in Study Groups a,b,c

\begin{tabular}{lccc}
\hline & \multicolumn{2}{c}{ Percent Changes } & \multirow{2}{*}{$\boldsymbol{P}$ Value } \\
\cline { 2 - 3 } & QSNYL-FEYL Group (n= 21) & QSNYL Group (n= 20) & 0.006 \\
\hline Visioface & $56.95(40.29)$ & $29.25(13.20)$ & 0.692 \\
Melanin nonlesional skin & $2.25(4.71)$ & $2.97(6.68)$ & $<0.001$ \\
Melanin lesional skin & $22.01(10.67)$ & $7.69(4.75)$ & 0.413 \\
Erythema nonlesional skin & $1.44(6.41)$ & $3.65(10.39)$ & 0.152 \\
Erythema lesional skin & $7.30(7.70)$ & $3.40(9.39)$ & \\
\hline
\end{tabular}

Abbreviations: QSNYL, Q-switched Nd:YAG laser; FEYL, fractional erbium:YAG laser.

a Data are presented as mean (SD).

${ }^{\mathrm{b}}$ Percentage changes are calculated as follows: [(value before treatment - value after treatment)/value before treatment] $\times 100$.

c Independent-samples t test was employed to compare values between two groups.

Table 3. Visioface Score, Melanin Content, and Erythema Intensity of Nonlesional and Lesional Skin after adjustment for Baseline Values ${ }^{\mathrm{a}, \mathrm{b}, \mathrm{c}}$

\begin{tabular}{lccc}
\hline & \multicolumn{2}{c}{ Adjusted Values After Treatment } & \multirow{2}{*}{$\boldsymbol{P}$ Value } \\
\cline { 2 - 3 } & QSNYL-FEYL Group (n= 21) & QSNYL Group (n= 20) & $<0.001$ \\
\hline Visioface mean score & $-2.83(0.485)$ & $-5.41(0.498)$ & 0.793 \\
Melanin, nonlesional skin & $155.87(1.91)$ & $155.15(1.96)$ & $<0.001$ \\
Melanin, lesional skin & $172.83(4.58)$ & $207.22(4.70)$ & 0.679 \\
Erythema, Nonlesional Skin & $326.13(6.97)$ & $322.51(6.12)$ & $<0.001$ \\
\hline Erythema, Lesional Skin & $333.08(6.40)$ & $365.31(6.58)$ & \\
\hline
\end{tabular}

Abbreviations: QSNYL, Q-switched Nd:YAG laser; FEYL, fractional erbium:YAG laser.

${ }^{a}$ Data are presented as mean (SD).

${ }^{\mathrm{b}}$ Percentage changes are calculated as follows: [(value before treatment - value after treatment)/value before treatment] $\times 100$.

cANCOVA test was used to compare the values between groups after adjustment for the baseline value for all variables.

a crossover, split-face clinical trial, Jeong et al compared the effect of 1064-nm QSEYL on melasma before and after treatment with topical triple combination (TTC; hydroquinone $5 \%$, tretinoin $0.05 \%$, and triamcinolone acetonide $0.1 \%$ cream) using patients' self-assessment, MASI score, and spectrophotometry. They reported better subjective results on the side that had received laser therapy after topical treatment. ${ }^{10}$ In another splitface study, Wattanakrai et al compared 5 session of weekly QSEYL and 2\% hydroquinone (laser group) with topical therapy (control) in Asian patients with dermal or mixed-type melasma. According to modified MASI score and photometric assay, they found significant improvement in the laser-treated side; however, they reported complications such as melasma recurrence, rebound hyperpigmentation, and hypopigmentation in follow-up. They concluded that such a laser therapy would induce temporary improvement with undesirable adverse effects. ${ }^{11}$

Kroon et al compared 8-week treatment with fractionated nonablative 1550-nm erbium glass laser (held every other week) with 8-week treatment with TTC. Although they found no difference between groups in GPA, selfsatisfaction was significantly higher in those treated with laser. They reported some adverse effects such as pain, burning sensation, and erythema after laser therapy; nevertheless, they recommended such a laser therapy as a safe and acceptable treatment for melasma. ${ }^{12}$ Wind et al compared fractionated nonablative $1550-\mathrm{nm}$ erbium laser with TTC and reported significantly lower GPA and satisfaction as well as high rate of hyperpigmentation (31\%) in the laser-treated sides and hence, did not recommend such a therapy for melasma. ${ }^{13}$

While laser therapies are associated with adverse effects such as burn and erythema and might induce post inflammatory hyperpigmentation, their usage in dermatology and cosmetic procedures is increasing. We did not employ MASI, GPA, or other subjective tools to assess the improvement; instead, we tried objective measures, which would provide less biased and more precise determination of changes in skin. Nonetheless, our study had some limitations.

First, the sample size was small because many of the patients did not intend to participate in a study that examined new treatment modalities. Most of the patients with melasma had attempted many other treatments with unfavorable results and were reluctant to undergo an aggressive laser therapy. Second, although we tried to use the devices that evaluate the lesions and their improvement objectively, these devices are operator-dependent and the degree of improvement might be affected by the spots chosen by the examiner. Nevertheless, a trained physician (first author), who was unaware of assigned treatment to each patient, performed all the measurements. Third, short-term follow-up after the last treatment session would affect the judgment on the long-lasting beneficial and adverse effects of therapy as well as changes in skin parameters. Fourth, we did not determine the depth of lesions. In fact, dermal lesions tend to be more resistant to administered treatments..$^{14}$ Using Wood lamp, dermal ultrasonography, 
or other modalities that can determine the depth of lesions would be more helpful in evaluating the effect of such therapies on dermal, mixed, or epidermal lesions. In conclusion, although both QSNYL-FEYL and QSNYL therapies in combination with topical therapy had favorable results, it seems that QSNYL-FEYL excels QSNYL in decreasing melanin content and brightening skin color. Nevertheless, studies with larger sample size and consideration for other factors that might affect the results, eg, drug history, pregnancy, and depth of lesions, are needed to determine the exact effect of such treatments. Melasma is a chronic disorder and longer follow-up period is needed to evaluate long-term effects of therapy on the course of disorder.

\section{Acknowledgments}

This study was performed with the financial support of Skin and Stem Cell Research Center, Tehran University of Medical Science, Tehran, Iran. We would like to express our sincere gratitude to Rasool Mohammadi, PhD candidate of epidemiology, for analyzing the data and for his helps in reporting the study results.

\section{Funding and Support}

This study was performed with the financial support of Skin and Stem Cell Research Center, Tehran University of Medical Science, Tehran, Iran.

\section{Ethical Considerations}

The protocol of the study conformed to the Helsinki declaration and was approved by Ethic Committee of our research center.

\section{Conflict of Interests}

The authors declared no conflict of interest with regard to materials and devices used in this study.

\section{References}

1. Ball Arefiev KL, Hantash BM. Advances in the treatment of melasma: a review of the recent literature. Dermatol Surg. 2012;38(7 Pt 1):971-984. doi:10.1111/j.15244725.2012.02435.x.

2. Moubasher AE, Youssef EM, Abou-Taleb DA. Q-switched Nd: YAG laser versus trichloroacetic acid peeling in the treatment of melasma among Egyptian patients. Dermatol Surg. 2014;40(8):874-882. doi:10.1097/ DSS.0000000000000065.

3. Yun WJ, Moon HR, Lee MW, Choi JH, Chang SE. Combination treatment of low-fluence 1,064-nm
Q-switched Nd: YAG laser with novel intense pulse light in Korean melasma patients: a prospective, randomized, controlled trial. Dermatol Surg. 2014;40(8):842-850. doi:10.1097/DSS.0000000000000057.

4. Lee DB, Suh HS, Choi YS. A comparative study of lowfluence 1064-nm Q-switched Nd:YAG laser with or without chemical peeling using Jessner's solution in melasma patients. J Dermatolog Treat. 2014;25(6):523-528. doi:10.3 109/09546634.2013.848261.

5. Morais OO, Lemos EF, Sousa MC, Gomes CM, Costa IM, Paula CD. The use of ablative lasers in the treatment of facial melasma. An Bras Dermatol. 2013;88(2):238-242. doi:10.1590/S0365-05962013000200009.

6. Niwa Massaki AB, Eimpunth S, Fabi SG, Guiha I, Groff W, Fitzpatrick R. Treatment of melasma with the 1,927$\mathrm{nm}$ fractional thulium fiber laser: a retrospective analysis of 20 cases with long-term follow-up. Lasers Surg Med. 2013;45(2):95-101. doi:10.1002/lsm.22100.

7. Kligman AM, Willis I. A new formula for depigmenting human skin. Arch Dermatol. 1975;111(1):40-48.

8. Nouri K, Bowes L, Chartier T, Romangosa R, Spencer J. Combination treatment of melasma with pulsed C02 laser followed by Q-switched alexandrite laser: a pilot study. Dermatol Surg. 1999;25:121-123.

9. Angsuwarangsee S, Polnikorn N. Combined ultrapulse $\mathrm{CO} 2$ laser and Q-switched alexandrite laser compared with Q-switched alexandrite laser alone for refractory melasma: split-face design. Dermatol Surg. 2003;29(1):59-64.

10. Jeong SY, Shin JB, Yeo UC, Kim WS, Kim IH. Low-fluence Q-switched neodymium-doped yttrium aluminum garnet laser for melasma with pre- or post-treatment triple combination cream. Dermatol Surg. 2010;36(6):909-918. doi:10.1111/j.1524-4725.2010.01523.x.

11. Wattanakrai P, Mornchan R, Eimpunth S. Low-fluence Q-switched neodymium-doped yttrium aluminum garnet $(1,064 \mathrm{~nm})$ laser for the treatment of facial melasma in Asians. Dermatol Surg. 2010;36(1):76-87. doi:10.1111/ j.1524-4725.2009.01383.x.

12. Kroon MW, Wind BS, Beek JF, et al. Nonablative 1550-nm fractional laser therapy versus triple topical therapy for the treatment of melasma: a randomized controlled pilot study. J Am Acad Dermatol. 2011;64(3):516-523. doi:10.1016/j. jaad.2010.01.048.

13. Wind BS, Kroon MW, Meesters AA, et al. Non-ablative 1,550 $\mathrm{nm}$ fractional laser therapy versus triple topical therapy for the treatment of melasma: a randomized controlled split-face study. Lasers Surg Med. 2010;42(7):607-612. doi:10.1002/lsm.20937.

14. Rajaratnam R, Halpern J, Salim A, Emmett C. Interventions for melasma. Cochrane Database Syst Rev. 2010(7):CD003583. doi:10.1002/14651858.CD003583. pub2. 